\title{
A smartphone-based colorimetric PET sensor platform with molecular recognition via thermally initiated RAFT-mediated graft copolymerization
}

\author{
Ceren Kaymaz Kuşçuoğlu ${ }^{\mathrm{a}}$, Hasan Güner ${ }^{\mathrm{b}}$, Meshude Akbulut Söylemez ${ }^{\mathrm{c}}$, Olgun Güven ${ }^{\mathrm{c}}$, \\ Murat Barsbay b,c,* \\ ${ }^{a}$ Institute of Science, Polymer Science and Technology Division, Hacettepe University, Beytepe, Ankara, 06800, Turkey \\ ${ }^{\mathrm{b}}$ UNAM, Institute of Materials Science and Nanotechnology, Bilkent University, Ankara, 06800, Turkey \\ ${ }^{\mathrm{c}}$ Department of Chemistry, Hacettepe University, Beytepe, Ankara, 06800, Turkey
}

\section{A R T I C L E I N F O}

\section{Keywords:}

Smartphone-based sensing

Colorimetric detection

PET-based sensor platform

\begin{abstract}
A B S T R A C T
In this work, we report a low-cost and easy-to-use molecularly-imprinted colorimetric sensor platform that can sense target analyte with high sensitivity and good selectivity. The platform has been examined for colorimetric detection and quantitation of a model textile dye, basic red 9 (BR9), by employing methacrylic acid (MAA), ethylene glycol dimethacrylate (EGDMA) and cumyl dithiobenzoate (CDB) as functional monomer, crosslinker and RAFT agent, respectively. Benzophenone (BP), a photoinitiator, was covalently immobilized on the surface of poly(ethylene terephthalate) (PET) and then thermally activated by homolytic cleavage to form radicals on the surface that initiate RAFT-mediated grafting of a thin recognition layer. The molecularly imprinted polymer (MIP) layer of about $100 \mathrm{~nm}$ showed excellent removal of $76.7 \%$ target dye in $10 \mathrm{~min}$ and high selectivity compared to other similar dyes with an imprinting factor of 10.31 in the competitive environment. The resulting MIP grafted PET substrate was efficiently used as recognition unit in a smartphone-based colorimetric detection method using a color adaptation algorithm for point-of-care applications. Based on the obtained performance, it is promising to use the method for the detection and quantification of various analytes with chromophores.
\end{abstract}

\section{Introduction}

The demand for synthetic dyes is increasing in many industries such as textile, leather, paper, packaging etc. [1]. About half of the produced dyes worldwide are lost in industrial effluents annually, and some of them are mixed into the surface and ground water resulting from process or urban activities [2]. Many studies revealed that textile dyes are highly toxic to living organisms and the nature [3-5]. Analytical results need to be obtained in the shortest time possible, however, conventional detection methods take a long time to yield an answer. The emergence of the latest technologies such as sensors based on molecularly imprinted polymers (MIPs) has demonstrated the potential to shorten the time span between sample uptake and results. However, many research and development studies are required to improve the selectivity and sensitivity of these sensors before they become real and reliable alternatives.

MIPs are three-dimensional synthetic networks having cavities recognizing the template in terms of shape and size [6,7]. MIPs have high selectivity against the target molecule, show high resistance to mechanical stress or temperature variations and can be effortlessly adapted to practical applications [8]. In order to obtain high binding capacity and efficiency, MIPs must have homogeneous distribution of recognition cavities [9]. The selected polymerization method plays a crucial role in providing uniformity to the MIP structure. It is reported that conventional free radical polymerization (FRP) methods result in heterogeneous distribution of binding sites and low affinity and imprinting efficiency besides irregular inner morphology and porosity in MIP structure [10]. In contrast, reversible-deactivation radical polymerization (RDRP) techniques reportedly increase the structural uniformity and improve binding properties [11-13]. Various MIP preparation methods such as bulk [14] and emulsion [15] polymerization, surface grafting [16], precipitation [17] or in-situ membrane synthesis [18] can be carried out via RDRP techniques. In particular, atom transfer radical polymerization (ATRP) $[16,19]$ and reversible addition fragmentation chain transfer (RAFT) polymerization [20-22] techniques have been widely used for the preparation of tailor-made MIPs under controlled conditions. It is reportedly known that, RAFT mechanism tailors the formation of network structure in a controlledmanner and improves the morphological uniformity of MIPs $[11,22,23]$.

\footnotetext{
* Corresponding author at: Department of Chemistry, Hacettepe University, Beytepe, Ankara, 06800, Turkey.

E-mail address: mbarsbay@hacettepe.edu.tr (M. Barsbay).
} 
An ideal sensor system should answer to expectations such as rapid response, ease of use, reusability and cost effectiveness. Colorimetric sensors are regarded among the promising analytical methods for the quantification and detection of a broad range of components such as volatile gases, proteins, heavy metals, pathogens, nucleic acids, etc. [24-27]. Particularly, combination of colorimetric sensors with digital imaging and software makes them very attractive and preferable [28]. MIPs particularly those prepared by grafting a thin layer on a support material are quite promising and can be used as recognition elements in sensor systems as they provide rapid and selective uptake of templates [19,25,29-32]. MIP-based colorimetric sensor systems are capable of recognizing the target molecules by monitoring them optically via the chromophore groups of the templates [33,34].

Benzophenone (BP) is of enduring interest as a photoactive tethering reagent that has been used to functionalize a variety of surfaces [35-37]. Photo-induced grafting of various monomers can be carried out through the radicals formed on the substrate surface as a result of homolytic cleavage of surface-attached BP under UV irradiation $[38,39]$. In a few studies, surface-immobilized BP was activated thermally to yield radicals on surface at high concentrations that may be used as initiating sites for grafting without interfering with the bulk properties of the substrate [40,41]. Activating BP thermally, instead of photochemically, is promising not only because it allows facile modification of surfaces and tailoring them to specific needs, but also as most of the common RAFT agents, especially dithiobenzoates, and many analytes with chromophore groups have low resistance against UV-radiation [42]. To the best of our knowledge, grafting a thin MIP layer on a substrate though thermal-activation of a chemically-immobilized photoactive reagent is a novel strategy. No systematic study has been published on preparation of a sensor platform via the approach applied in this study. The facile synthesis route used here based on a model template, BR9, is quite promising for the detection/quantification of a variety of analytes with chromophores in different matrices.

\section{Experimental}

\subsection{Materials}

Ethylene glycol dimethacrylate (EGDMA), benzophenone (BP), 4,4'Azobis-4-cyanopentanoic acid (ACPA), acetic acid, ethanol, methanol, sodium hydroxide $(\mathrm{NaOH})$, hydrogen peroxide $\left(\mathrm{H}_{2} \mathrm{O}_{2}\right)$, hydrochloric acid ( $\mathrm{HCl}$ ), basic red 9 (BR9) were purchased from Sigma Aldrich. Ethanol, methacyrlic acid (MAA) and dioxane were purchased from Merck. PET films were obtained from Toray Plastics Europe Lumirror Company from United Kingdom. Cumyl dithiobenzoate (CDB) was employed as the RAFT agent and purchased from Sigma Aldrich (see Fig. 1 for the chemical structures). 4 x Philips CLEO (15 W) lamps used as UV irradiation source. Methyl violet (MV), methylene blue (MB) and disperse orange 11 (DO11) were employed as competitive dyes and purchased from BDH, Fisher and Dr. Ehrenstarfer GmbH, respectively.

\subsection{Preparation of MIP grafted PET surfaces}

\subsubsection{Hydrolysis}

PET films were cut into pieces with ca. $2 \mathrm{~cm} \times 2 \mathrm{~cm}$ dimensions and washed with methanol/water mixture (50:50, v/v) and allowed to dry prior to use. The clean films were hydrolyzed in $2 \mathrm{M} \mathrm{NaOH}$ solution at $70{ }^{\circ} \mathrm{C}$ for $90 \mathrm{~min}$. The carbon atom of the ester bond of PET is attacked by the unpaired electrons on the oxygen atom of $\mathrm{NaOH}$, yielding carboxyl and alcohol end groups at the point where this attack ends with chain breaks in PET structure [43]. The films were then kept in acetic acid/water solution $(50: 50, \mathrm{v} / \mathrm{v})$ for $15 \mathrm{~min}$ at $70^{\circ} \mathrm{C}$ and washed with distilled water to remove residual $\mathrm{NaOH}$ from surface and dried in vacuum.

\subsubsection{Oxidation}

In order to increase the carboxyl group concentration for better uptake of $\mathrm{BP}$, alcohol groups at the chain ends were converted into carboxyl groups by treatment with $0.6 \mathrm{M} \mathrm{H}_{2} \mathrm{O}_{2}$ solution for $300 \mathrm{~min}$ under UV radiation at a distance of $10 \mathrm{~cm}$ from the lamp to the film [44]. The $\mathrm{pH}$ of the solution was adjusted to 3 using $0.1 \mathrm{M} \mathrm{HCl}$. After oxidation the films were washed with distilled water and allowed to dry. In order to determine the amount of - $\mathrm{COOH}$ groups formed on the PET surface, carboxylic acid groups were measured via a titration method [45]. Briefly; hydrolyzed/oxidized PET films with an area of about $1 \mathrm{~cm}^{2}$ were immersed into $0.5 \mathrm{mM}$ toluidine blue (TBO) solution and the $\mathrm{pH}$ was adjusted to 10 using $0.1 \mathrm{M} \mathrm{NaOH}$. The ionic complex formation between the cationic TPO dye and the anionic -COO- groups was allowed to proceed for $6 \mathrm{~h}$ at room temperature. At the end of the standby time the excess TBO was rinsed by $0.1 \mathrm{mM} \mathrm{NaOH}$ solution. For the desorption of TBO molecules bound on PET films, the films were incubated in $5 \mathrm{~mL}$ of $50 \%$ acetic acid solution for $20 \mathrm{~min}$. The absorbance of desorbed TPO was measured at $633 \mathrm{~nm}$. It is assumed that TBO is bound to $\mathrm{COOH}$ with 1:1 stoichiometric ratio [46]. The amount of carboxyl groups on the films was determined as $1.1 \pm 0.088 \mathrm{nmol} / \mathrm{cm}^{2}$ (average of five measurements) using the calibration curve given in Fig. S1 of Supporting Information.

\subsubsection{BP immobilization}

The oxidized PET films with surface of $2 \mathrm{~cm}^{2}$ were soaked in $\% 10 \mathrm{BP}$ in dioxane $(\mathrm{w} / \mathrm{w})$. The mixture was sonicated for $10 \mathrm{~min}$, and then UV<smiles>CC(C)(SC(=S)c1ccccc1)c1ccccc1</smiles>

CDB<smiles>O=C(c1ccccc1)c1ccccc1</smiles>

BP<smiles></smiles>

BR9<smiles>CC(C)(CCC(=O)O)NNC(C)(N)CCC(=O)O</smiles>

ACPA<smiles>C=C(C)C(=O)O</smiles>

MAA<smiles>C=C(C)C(=O)OCCOC(=O)C(=C)C</smiles>

EGDMA

Fig. 1. Chemical structures of the RAFT agent (CDB), surface-immobilized photoactive reagent (BP), model template (BR9), thermal initiator (ACPA), monomer (MAA) and cross-linker (EGDMA). 
irradiated at room temperature for $60 \mathrm{~min}$. The distance between the UV lamp and the films was $10 \mathrm{~cm}$. After the reaction, the films were removed from the BP solution, immersed in dioxane for $5 \mathrm{~min}$ to remove the excess of BP and transferred into the reaction tubes containing polymerization solution.

\subsubsection{MIP layer formation via RAFT-mediated graft copolymerization from} PET surfaces

Grafting of molecularly imprinted polymers from the BP-immobilized PET surfaces was carried out thermally at $75^{\circ} \mathrm{C}$ via RAFTmediated graft copolymerization in the presence of monomer (MAA), RAFT agent (CDB), crosslinker (EGDMA), thermal initiator (ACPA) and template molecule (BR9). Along with the BP-immobilized PET films a free-radical initiator (ACPA) was added to the solution to provide better control of the growth of the chains initiated form the surface [47]. Polymerization solution was prepared in accordance with certain ratios of monomer, crosslinker, RAFT agent, template and thermal initiator in a total of $10 \mathrm{~mL}$ solution using ethanol as solvent. MAA and BR9 were added into ethanol first, and the solution was kept at ambient temperature for $1 \mathrm{~h}$ to ensure the formation of the complex between the template and the monomer. The ratios of MAA/BR9 and MAA/EGDMA were set at $4 / 1$ and $1 / 2$, respectively. The ratio of ([MAA] $+[$ EGDMA] $) /[\mathrm{CDB}]$ was adjusted to the optimum value of 1330 [11]. The molar ratio between CDB and ACPA was kept either as 2 or 10. BPimmobilized PET films were then immersed into the polymerization solution and the content was sealed and purged with nitrogen for $10 \mathrm{~min}$. The reaction took place in a shaking bath $(100 \mathrm{rpm})$ at $75^{\circ} \mathrm{C}$ for $3.5 \mathrm{~h}$. Polymerizations were terminated before the gel formation by opening reaction vials to atmosphere and cooling down to room temperature. MIP grafted PET films were removed from the polymerization solution, and the solution was precipitated in 10 fold excess of diethyl ether to yield free, i.e. non-grafted, poly(methacrylic acid-co-ethylene glycol dimethacylate), p(MAA-co-EGDMA), formed simultaneously in grafting medium. The films were washed with deionized water, ethanol and acetone to remove the template from the MIP layer. The solvent was changed periodically until no BR9 could be identified in the rinsing solution via UV analysis. Finally, the MIP grafted PET films were washed with $1 \mathrm{M} \mathrm{NaOH}$ solution for $10 \mathrm{~min}$ and then with deionized water until a neutral $\mathrm{pH}$ was obtained. The whole procedure applied for preparation of the MIP grafted PET surfaces was summarized in Fig. 2. The non-imprinted polymer (NIP) grafted PET films were prepared by applying the same grafting procedure except the addition of the BR9 template.

\subsection{Evaluation of BR9 binding capacity}

Standard aqueous BR9 solutions with various concentrations (0.1-100 ppm) were used in dye uptakes. PET films with pre-measured surface areas and weights were placed in $1.0 \mathrm{~mL}$ of dye solution for $10 \mathrm{~min}$. The amount of BR9 before and after incubation period was determined using UV-Vis spectrophotometer at the specific wavelength of $540 \mathrm{~nm}$. All the analyses were repeated thrice. The adsorption isotherms were evaluated using the data attained. The calibration curve used was given in Fig. S2. The limit of detection (LoD) value was determined as $0.04 \mathrm{ppm}$ using Eq. S1 of Supporting Information. The kinetics of adsorption was carried out in $0.5 \mathrm{ppm}$ BR 9 solution by varying the incubation time (1-60 $\mathrm{min})$.

Selectivity of MIPs for BR9 was evaluated by performing the BR9 binding studies in the presence of analogue dyes; methyl violet (MV), methylene blue (MB) and disperse orange (DO11). The structures of the competitive dyes along with their maximum absorption wavelengths were demonstrated in Fig. S3. The molar concentration of each dye was $1.00 \mathrm{ppm}$, incubation time was $10 \mathrm{~min}$.

In order to evaluate the reusability, BR9-free imprinted PET was incubated in 0.50-ppm BR9 solution for $10 \mathrm{~min}$. After determining the binding capacity, the MIPs were washed as descried before. The BR9 uptake/removal cycle was repeated 10 times in the same way.

\subsection{UV-vis spectrophotometry}

The amount of target molecules in the solutions were assigned by UV-vis Varian Cary100 spectrophotometer. Measurements were performed between $190-800 \mathrm{~nm}$ wavelength at room temperature.

\subsection{Attenuated total reflectance fourier transform infra-red (ATR-FTIR) spectroscopy}

FTIR spectra were recorded in attenuated total reflection (ATR) mode by Perkin Elmer Spectrum One model spectrometer. Spectra were obtained by 32 scans with a $4 \mathrm{~cm}^{-1}$ resolution.

\subsection{X-ray photoelectron spectroscopy (XPS)}

X-ray photoelectron experiments were performed using monochromatized $\mathrm{Al} \mathrm{K} \alpha \mathrm{X}$-ray source of Thermo spectrometer. Survey and region scans were recorded with $30 \mathrm{eV}$ and $200 \mathrm{eV}$ pass energy, respectively. All samples were analyzed at a take-off angle of $90^{\circ}$. Surface elemental composition was determined using an X-ray spot size of $400 \mu \mathrm{m}$ by varying the energy between $0-1000 \mathrm{eV}$. Binding energies (BEs) were referenced to the $\mathrm{C} 1 \mathrm{~s}$ hydrocarbon peak at $285 \mathrm{eV}$.

\subsection{Atomic force microscopy (AFM)}

Changes on the surface morphology were investigated using Bruker Nanoscope 9 atomic force microscopy in tapping mode in air.

\subsection{Ellipsometry}

We acquired the ellipsometric angles $\psi$ and $\Delta$ using J.A. Woollam Company V-VASE rotating analyzer ellipsometer at three angles of incidence $\left(\phi_{0}=50^{\circ}, 55^{\circ}\right.$, and $\left.60^{\circ}\right)$ over the spectral range of $400-1200 \mathrm{~nm}$. Optical modeling and data analysis were performed using the Woollam Company software package.

\subsection{Contact angle (CA)}

The water CA values of pristine, hydrolyzed, oxidized, BP immobilized, and MIP grafted PET surfaces were measured at room temperature using a Kruss DSA 100 goniometer system. The CA measurements were performed using the Young-Laplace method of the Drop Shape Analysis program (Krüss Company).

\subsection{Investigation of colorimetric sensor performance}

\subsubsection{Measurement protocol}

The MIP and NIP grafted PET films were submerged into a range of standard BR9 solutions for $10 \mathrm{~min}$, washed with deionized water for $5 \mathrm{~s}$ for surface clearance, and dried in a vacuum chamber at room temperature. The PET samples were then placed on a blank white paper all together. The images of the PET samples were taken by a Samsung Galaxy Note 5 smartphone camera at a distance of $36 \mathrm{~cm}$ and an angle of 30 degrees with respect to the surface normal (please see Image Capture Configuration, Fig. S4 of Supplementary Information). The images were captured under the illumination of only smartphone's built-in LED flash light with automatic camera settings. All the images were saved in JPEG format with a resolution of $5312 \times 2988$ pixels.

\subsubsection{Image processing}

Quantification of color changes are commonly performed by using color components of different color spaces in smartphone-based colorimetry applications [48]. It is critical to identify the appropriate color space in order to achieve the best available sensing performance [49]. 


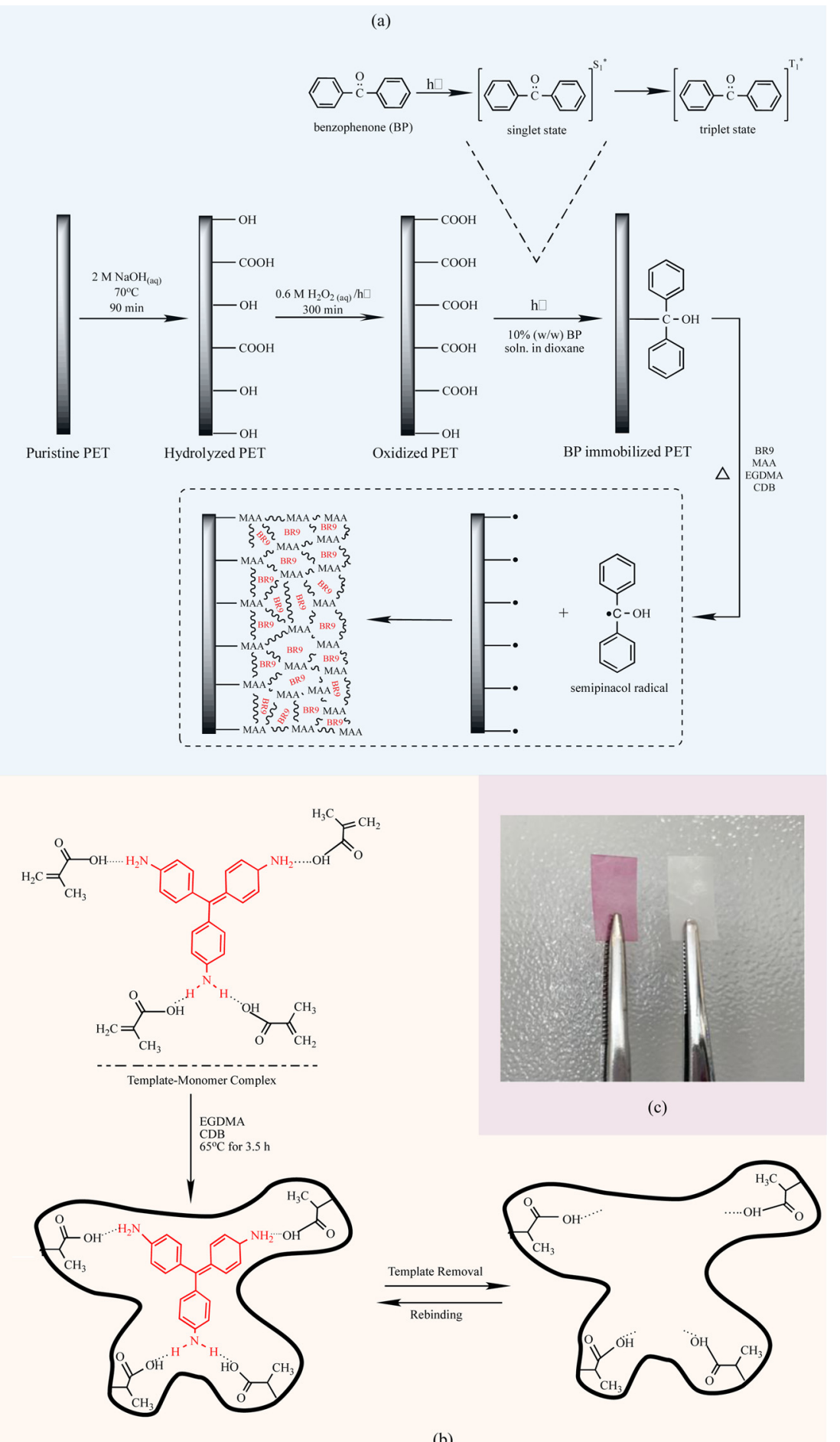

(b)

Fig. 2. (a) Schematic representation of the steps applied for the preparation MIP-grafted PET surface, (b) representative expression of the formation of templatespecific cavities, (c) photo of the MIP-grafted PET films before (magenta) and after (white) the template removal.

Previously, paper-based colorimetric assays have been reported that use RGB [50,51], HSV [48,49], CMYK [52,53] and CIE 1931 [54,55] color spaces. Here, both HSV and CMYK color spaces are employed for colorimetric analysis and compared in terms of sensor performance metrics.

The image files were preprocessed and analyzed in Matlab programming environment. First, the nonlinear sRGB images as originally stored in the smartphone memory were linearized through the following equations $[54,55]$ :
$C^{\prime}{ }_{s r g b}=C_{s r g b} / 255$

$C_{l}=\left(\frac{C^{\prime}{ }_{s r g b}+0.055}{1.055}\right)^{2.4}$

where $C_{\text {srgb }}$ stands for R (red), G (green) and B (blue) channels in the sRGB color space, and $C_{l}$ represents the linearized R, G and B values. Next, the linearized RGB values were normalized by the estimated white background image using Eq. (3) in order to correct for uneven distribution of illumination on the sample set [48]. 
$C_{c}=C_{l} / C_{w}$

where $C_{c}$ and $C_{w}$ correspond to the R, G and B channel intensities of the corrected and estimated white background images, respectively. The estimated white background images were obtained by setting $5 \times 4$ reference points on the blank white paper background distributed over the entire area of the sample image followed by second-order bivariate polynomial regression. The corrected RGB values were then converted into the HSV and uncalibrated CMYK values where $S$ and $M$ channel intensities were utilized as sensor signals. The $\mathrm{H}$ (hue), $\mathrm{S}$ (saturation) and $\mathrm{V}$ (value) values were calculated by the following transformations [56]:

$$
H=\left\{\begin{array}{c}
0 \text { if max }=\text { min } \\
60^{\circ} \frac{G_{c}-B_{c}}{\max -\min } \text { if } \max =R_{c} \text { and } G_{c} \leq B_{c} \\
60^{\circ} \frac{G_{c}-B_{c}}{\max -\min }+360^{\circ} \text { if } \max =R_{c} \text { and } G_{c}>B_{c} \\
60^{\circ} \frac{B_{c}-R_{c}}{\max -\min }+120^{\circ} \text { if } \max =G_{c} \\
60^{\circ} \frac{B_{c}-R_{c}}{\max -\min }+240^{\circ} \text { if } \max =B_{c}
\end{array}\right.
$$

$$
S=\left\{\begin{array}{c}
0 \text { if } \max =\min \\
\frac{\max -\min }{\max +\min } \text { if } V \leq 0.5 \\
\frac{\max -\min }{2-(\max +\min )} \text { if } V>0.5
\end{array}\right.
$$

$V=\max$

where max and min denote maximum and minimum values of $R_{c}, G_{c}$ and $B_{c}$, respectively.

The uncalibrated C (cyan), M (magenta), Y (yellow) and K (key) values were calculated using the following equations:

$$
\begin{aligned}
& C=\max -R_{c} \\
& M=\max -G_{c} \\
& Y=\max -B_{c} \\
& K=1-\max
\end{aligned}
$$

Following the color space conversions, square shaped region of interests $(50 \times 50$ pixels) were defined at the central regions of each sample and channel intensities were spatially averaged over the enclosed area. The averaged S and M channel intensities of the NIP samples associated with each analyte concentration were subtracted from those of the MIP samples in order to eliminate the non-specific binding effects.

\subsection{Binding studies in real samples}

Real water samples were collected near Ankara (Susuz lake, Beytepe pond, Kuzoglu creek and tap water). A water sample labelled 'wastewater' was obtained from a manufacturer of textile chemicals, Rudolf $\mathrm{GmbH}$ (Bursa, Turkey). Water samples were filtered through a $0.45 \mu \mathrm{m}$ Nylon filter and stored in the dark at $4{ }^{\circ} \mathrm{C}$. Prior to analysis, the samples were spiked with BR9 at a concentration of $1.0 \mathrm{ppm}$. MIP grafted PET films with pre-measured surface areas and weights were placed in $1.0 \mathrm{~mL}$ of real water samples for $10 \mathrm{~min}$. The amount of BR9 before and after incubation period was determined using UV-Vis spectrophotometer at the specific wavelength of $540 \mathrm{~nm}$.

\section{Results and discussion}

\subsection{Grafting of MIPs from the surface of PET}

Preparation of molecularly-imprinted PET surfaces was carried out in four steps: hydrolysis and oxidation of PET, immobilization of BP and graft copolymerization. Korolkov et al. reported that oxidized surfaces containing carboxyl groups can adsorb almost twice amount of BP compared to non-oxidized ones [44]. There is a straightforward relationship between the immobilized BP concentration on a surface and grafting yield $[57,58]$. Therefore, the first two pre-functionalization steps were critical in terms of the amount of immobilized BP on PET. It is well recognized that photochemically generated triplet state of $\mathrm{BP}$ can abstract a labile hydrogen atom from almost all polymers with the formation of radicals on surface at high concentrations [35]. The resulting radicals combine with monomers in the polymerization medium and graft copolymerization takes place upon UV irradiation [31,35,59]. This photoinduced grafting method has been used to permanently attach various polymers to a broad selection of surfaces for many different applications, such as patterned sheets [60], biosensors [61], organic semiconductors [62] etc. A series of experiments have shown that excited benzophenone is chemically immobilized at the surface upon UV irradiation in the absence of monomer, again by abstracting a hydrogen atom from the surface (see Fig. 2a). Yang and Ranby chemically bonded three photoinitiators (benzophenone, xanthone, and 9-fluorenone) on low-density polyethylene films by UV irradiation, and performed graft copolymerization from the films by either photochemically or thermally activating the photoinitiators [40]. All three of photoactive reagents were homolytically cleaved from the surface to yield surface radicals and semipinacol radicals through either photo- or thermal- activation. The surface radicals initiated free-radical graft copolymerization, whereas the semipinacol radicals were relatively less reactive and not prone to initiate homopolymerization. Wang et al. covalently immobilized BP on vulcanized acrylonitrile butadiene rubber (NBR) particle surface by UV irradiation [41]. When they contacted BP-immobilized NBR particles with styrene above $80^{\circ} \mathrm{C}$, graft copolymerization did indeed start from the surface of the particles. Furthermore, they were able to prepare composite core-shell particles by adding a second monomer (acrylamide or $\mathrm{N}$-vinyl pyrrolidone) through the thermal cleavage of weak $\mathrm{C}-\mathrm{C}$ bonds at the surface-attachment points of semipinacol end-groups.

As can be seen in Fig. S5 of supplementary information, both the RAFT agent and BR9 dye decompose under direct UV-radiation. Most of the common RAFT agents, especially dithiobenzoates, have low resistance against UV [42]. Therefore, preparation of MIP layer on PET cannot been carried out under direct UV light. Instead, we have subjected BP-immobilized PET substrate to graft copolymerization in a thermal-activation approach. BP molecules were thermally cleaved from the parent polymer and the surface radicals initiated grafting of MIP layer, i.e. poly(methacrylic acid-co-ethylene glycol dimethacrylate), poly(MAA-co-EGDMA).

As can be seen in the FTIR spectra presented in Fig. S6 of supporting information, intensity of the carbonyl peak, $\mathrm{C}=\mathrm{O}$, at $\sim 1720 \mathrm{~cm}^{-1}$ increased after the oxidation process. A slight $-\mathrm{OH}$ peak at $\sim 3400 \mathrm{~cm}^{-1}$ indicates the enrichment of the surface in hydroxyl groups. The immobilization of BP on PET surface caused a broad significant peak at $3440 \mathrm{~cm}^{-1}$ assigned to the $\mathrm{OH}$ groups of semipinacols. Contact angle (CA) measurements presented in Fig. S7 confirmed these changes by the decrement of CA from $85.51^{\circ}$ to $63.92^{\circ}$ following the oxidization step. Immobilization of BP to the surface yields even more hydrophilic character due to increased - $\mathrm{OH}$ groups as indicated by the decrease of CA to $55.75^{\circ}$. Lowering of CA upon the grafting of MIP layer hence increasing hydrophilicity are due to presence of - $\mathrm{COOH}$ groups in poly(MAA-co-EGDMA) structure.

In a well-designed RAFT polymerization the molar ratio between RAFT agent and initiator is very crucial for optimal control. The molar ratio of the RAFT agent and initiator should be adjusted to minimize the number of initiator derived chains [42]. It is reported that the semipinacol radicals formed after cleaving of surface-attached BP are not prone to initiate homopolymerization [40]. The amount of free- ACPA added to the polymerization medium is therefore crucial. We have 


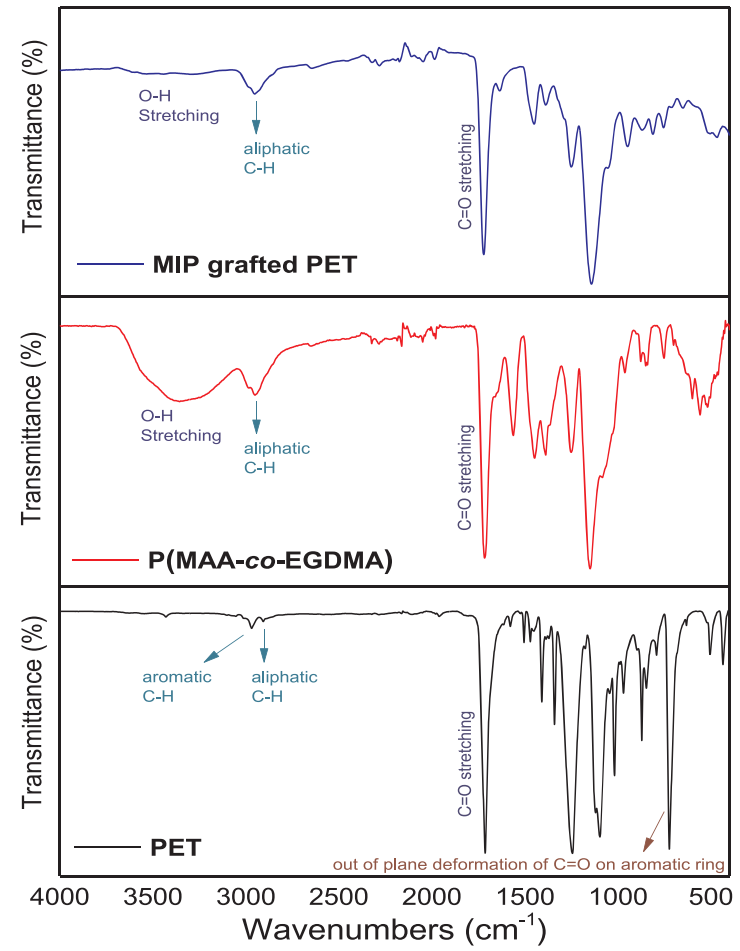

Fig. 3. ATR-FTIR spectra of PET, poly(MAA-co-EGDMA) and MIP-grafted PET. tested two different molar CDB/ACPA ratios of $2 / 1$ and $10 / 1$ by examining the adsorption capacities of the resultant MIPs as seen in Fig. S8. A significant difference was observed in binding capacities; the MIPs prepared using 2/1 ratio was significantly more efficient, indicating the formation of template-specific cavities either in higher quantities or selectivity. Therefore, $\mathrm{CDB} / \mathrm{ACPA}$ ratio of $2 / 1$ was chosen for the rest of the study.

\subsection{Characterization of the MIP-grafted PET surfaces}

The ATR-FTIR spectra of pristine and MIP grafted PET samples along with that of bulk MIP matrices (poly(MAA-co-EGDMA), see Fig. S9) are shown in Fig. 3. The main absorption bands of pristine PET are in very good agreement with literature data [63]. Upon surface-imprinting poly(MAA-co-EGDMA) matrices on PET surfaces cause the appearance of a small O-H stretching band centered at ca. $3200 \mathrm{~cm}^{-1}$. Aromatic C-H stretching peak of pristine PET observed at $2972 \mathrm{~cm}^{-1}$ disappears due to coverage of surface by aliphatic MIP functionalities. Carbonyl, $\mathrm{C}=\mathrm{O}$, stretching band at about $1715 \mathrm{~cm}^{-1}$ is common for all spectra. One distinct difference observed in the spectrum of PET is the disappearance of strong out of plane deformation peak of two carbonyl substituents on the aromatic ring centered at $729 \mathrm{~cm}^{-1}$. This shows that although the $\mathrm{C}=\mathrm{O}$ peak is common, chemical environment of the carbonyl groups in pristine PET is not the same with those existing in MIP grafted PET, clearly indicating the surface coverage of PET with poly (MAA-co-EGDMA) matrixes.

Topographic images of pristine, oxidized and imprinted PET films were obtained by AFM analysis (Fig. 4). The image of the pristine PET film came in sight as a smooth surface with low roughness (Rq) value of
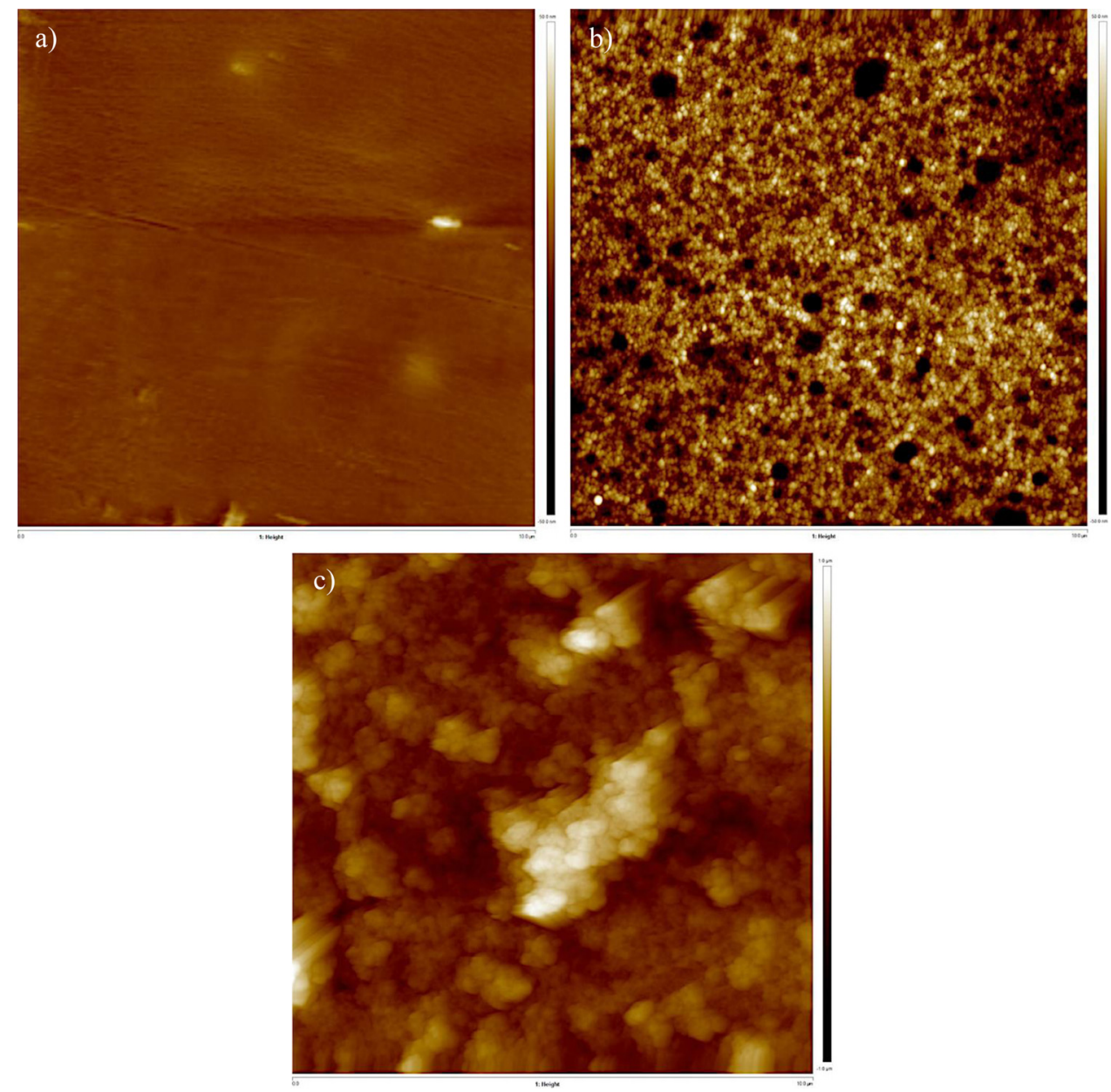

Fig. 4. AFM topographic images of (a) pristine PET, (b) oxidized PET and (c) MIP grafted PET, polymerization time: $3.5 \mathrm{~h}$. 


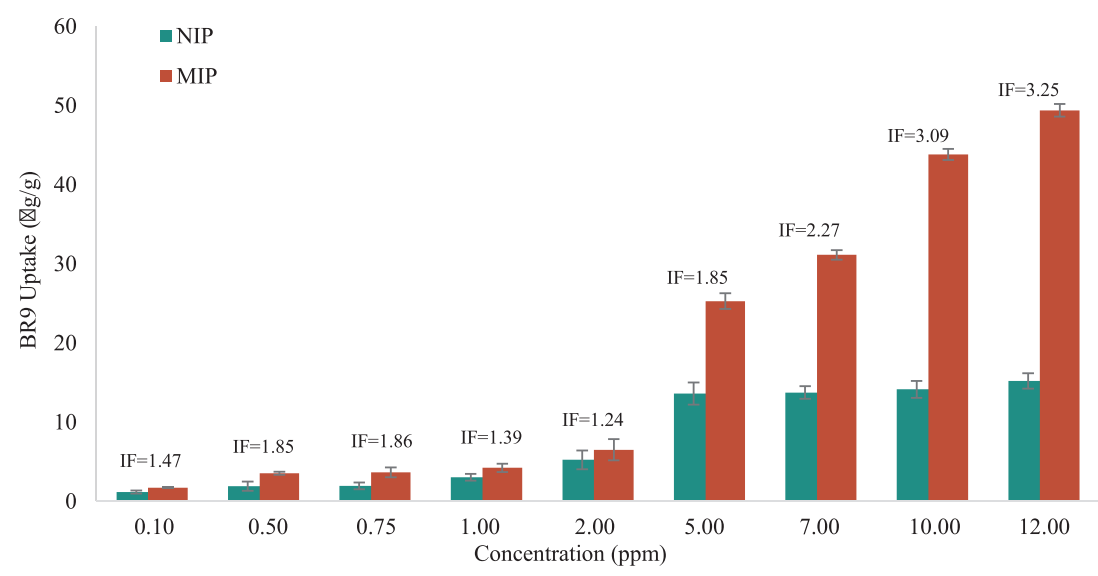

Fig. 7. Binding capacities of MIP and NIP grafted PET films at various feed concentrations $(n=3)$ and imprinting factor (IF) calculated for each feed concentration.

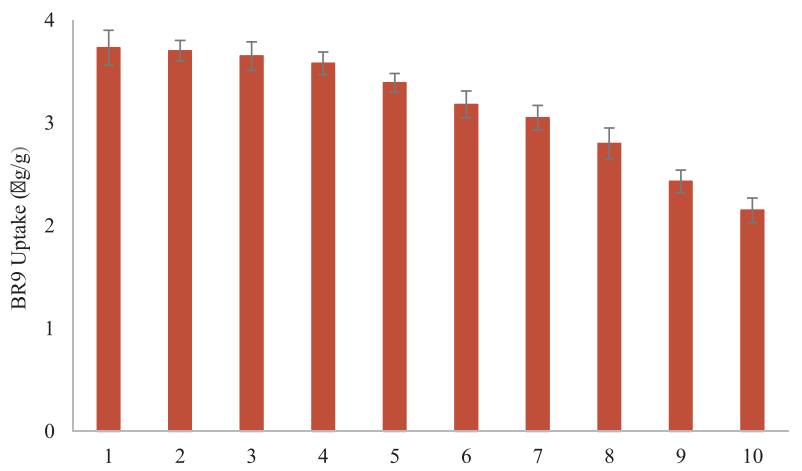

Fig. 8. Binding performance of the same MIP system after each use for ten cycles. BR9 concentration: $0.5 \mathrm{ppm}$, incubation time: $10 \mathrm{~min}, \mathrm{n}=3$.

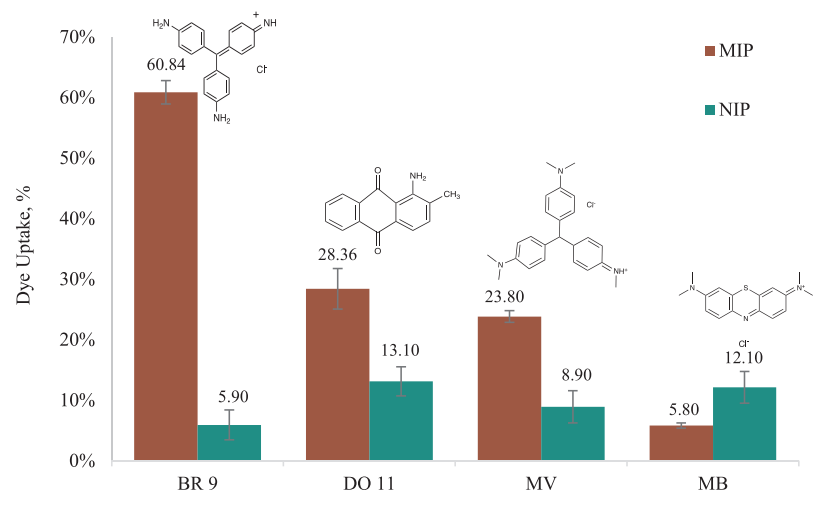

Fig. 9. Selectivity of BR9 imprinted polymer grafted PET films against DO11, MV and MB $(\mathrm{n}=3)$.

Langmuir isotherm is the most commonly used binding model for adsorption studies [67,68], it cannot be applied to imprinted polymers due to logarithmic distribution of the binding sites in multilayers $[69,70]$. Freundlich isotherm allows a better examination of different binding sites existing in imprinted polymer networks compared to Langmuir [71]. Values obtained from Freundlich isotherm along with kinetic parameters to be discussed later are summarized in Table 1. Heterogeneity of binding regions $(m)$ can be measured using the Freundlich binding model. This heterogeneity index ranges from 0 to 1 and increases as homogeneity increases in a system and the exact value of 1 illustrates the ideal homogeneity [72,73]. The value of $m$ was determined as 0.99 as can be seen in Table 1. The most important reason for applying the RAFT polymerization was to provide uniformity across the MIP network and it was indicated by the $m$ value that RAFT mechanism effectively ensures the achievement of a well-ordered structure. This result is in agreement with previously published works $[11,67,70,74,75]$.

Kinetics of adsorption is important since it explains the adsorption behavior and controls efficiency. As it can be seen in Fig. 6, adsorption takes place very quickly in the first $5 \mathrm{~min}$, then reach to equilibrium within 10 min. Such a fast binding kinetics is crucial for sensor applications and indicates ease of accessibility of template molecule to the binding sites. Evaluation of the first and pseudo second order kinetic models given in Table S1 of Supporting Information yield equilibrium adsorption $\left(\mathrm{q}_{\mathrm{e}}\right)$ and rate constant $(\mathrm{k})$ values. As it is evident in Table 1 , the data fit better the pseudo second order kinetic model with a correlation coefficient of 0.99 (Fig. S12). This indicates that rate-limiting step is controlled by chemical affinity between MIP and BR9, not by diffusion [76,77].

\subsubsection{Comparison of the performances of MIPs and NIPS}

Non-imprinted polymer (NIP) grafted PET films were prepared by applying the same grafting procedure in the absence of template molecule, BR9. As it is evident in Fig. 7, the MIP exhibited significantly higher adsorption capacity in comparison with the NIP. The higher affinity across the whole concentration range observed for MIP surfaces indicates that the interactions with BR9 were based on molecular recognition, whereas only non-specific interactions prevailed between NIP and BR9 [78]. The imprinting factor (IF) is defined as the ratio of binding of template molecule on MIP to binding on NIP, and it is a measure showing the specificity of interactions between the imprinted polymer and the template molecule [79]. As seen in Fig. 7, IF values increase with increasing feed concentration for MIP system, indicating that there are sufficient specific binding sites in the MIP structure that are easily accessible by the target molecules. For the NIP system, on the other hand, non-specific binding sites are fully occupied at a low feed concentration of $5.0 \mathrm{ppm}$ beyond which adsorption equilibrium is reached as no target molecule specific cavities are present in its structure.

\subsubsection{Reusability study}

For many applications, the reusability of a sensor material is an important parameter. The potential for multiple uses of the MIP grafted PET films was tested for ten identical cycles with the results summarized in Fig. 8. As it is apparent, there is not a significant decrease until the fifth cycle after which the binding capacity gradually decreases. The results clearly showed that the BR9-imprinted films could be used for several times (up to 5) without losing their adsorption capacities significantly.

\subsubsection{Selectivity study}

The selectivity studies were carried out in existence of three analogue dyes; disperse orange 11 (DO 11), methyl violet (MV), and 
(a)

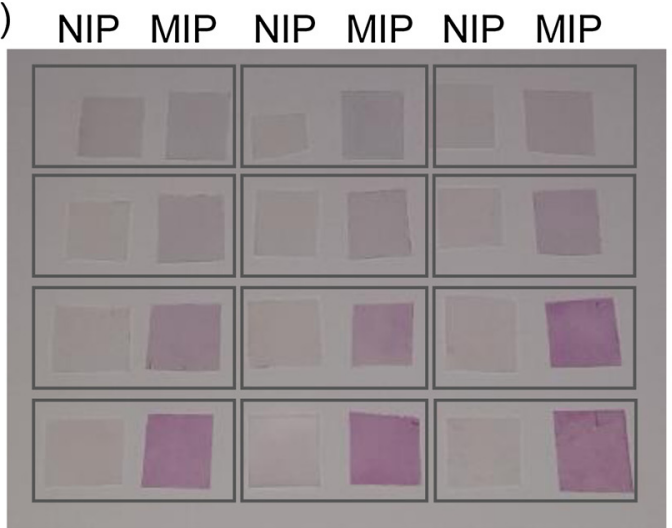

(c)

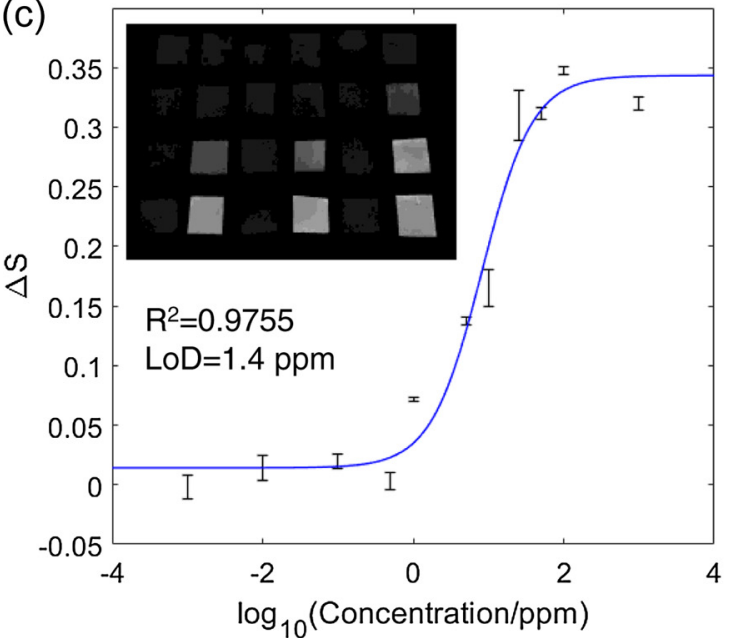

(b)

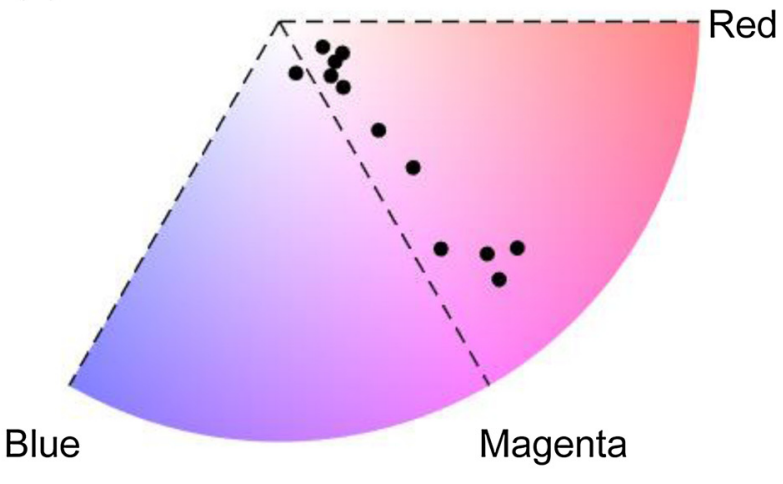

(d)

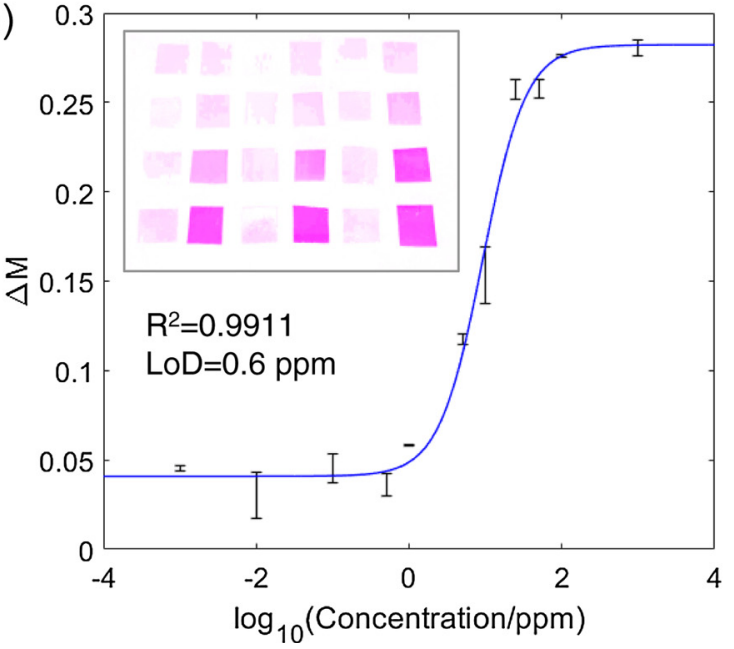

Fig. 10. Colorimetric analysis results. (a) Photograph of a set of MIP and NIP grafted PET samples. BR9 concentrations are $0,10^{-3}, 10^{-2}, 0.1,0.5,1,5,10,25,50,10^{2}$ and $10^{3} \mathrm{ppm}$ from top left to bottom right. (b) $\mathrm{H}$ and $\mathrm{S}$ channel intensities of the color-corrected MIP sample images are represented on the HSV color wheel. V channel intensities are taken as 1 for visual purposes. Dashed lines indicate the coordinates of blue, magenta and red colors. Calibration curves of the colorimetric sensor obtained by sigmoid function fitting to the experimental $\Delta S$ (c) and $\Delta M$ (d) values. Error bars denote twice the standard deviation of the experimental data. Inset figures show normalized S (c) and false-colored magenta of $M(d)$ channel images of a sample set. (For interpretation of the references to colour in this figure legend, the reader is referred to the web version of this article).

Table 2

Analytical specifications of the sensor system for colorimetric detection of BR9 dye.

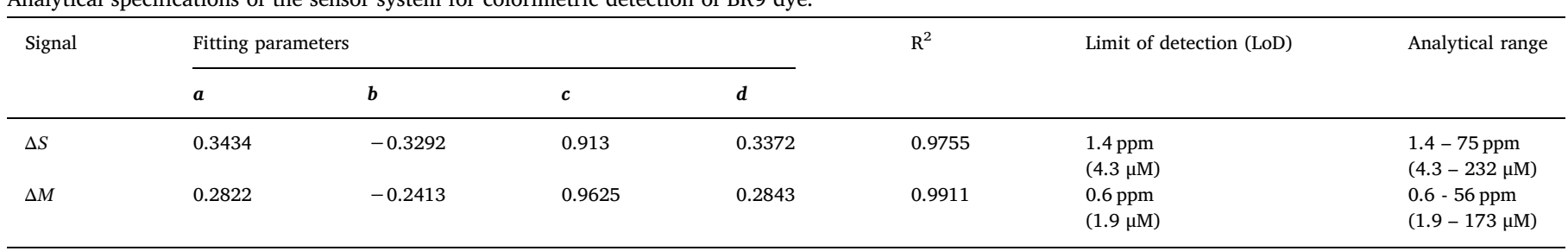

methylene blue (MB). As seen in Fig. 9, the highest IF value (the ratio of binding capacities of MIPs to NIPs) was obtained for BR9 as 10.31. The IF values were $2.16,2.67$, and 0.47 for the competitive dyes of DO 11 , $\mathrm{MV}$, and MB, respectively. The selectivity of MIPs is related to the complementarity of functional groups, sizes and shapes between template and recognition cavities. The adsorption of other dyes can be explained with the similarities in molecular shape, functional groups and non-specific interactions [80]. In the competitive environment, all dyes have similar affinity in filling non-specific regions, while the specific cavities in MIP only recognize and selectively attach the target dye, yielding an increase in IF value. The selectivity coefficient (k) is generally used to evaluate the degree of selectivity of MIPs and NIPs towards the target molecule and it can be determined as expressed in
Table S2 [81]. The results listed in Table S3 indicate that the selectivity of MIPs towards BR9 is 4.64, 5.31 and 25.22 times higher than those towards DO 11, MV and MB, respectively. We believe that RAFT polymerization was crucial in obtaining high performance in BR9 recognition by providing a uniform distribution of specific cavities and an easy accessibility to the recognition sites [82].

\subsubsection{Investigation of the performance of colorimetric sensors}

The test samples including both MIP and NIP grafted PET films were evaluated within a concentration range of $0-1000 \mathrm{ppm}$. All samples were placed on a white background and imaged by a smartphone camera (Fig. 10a). Image capture process was repeated three times under the same conditions. Image set was preprocessed through the 


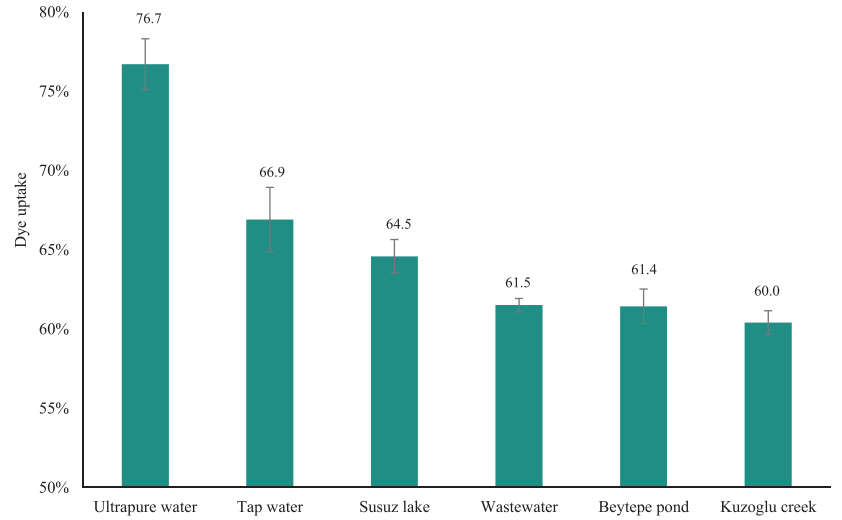

Fig. 11. Binding performance of MIP grafted PET films in real water samples. BR9 spiked concentration: $1.0 \mathrm{ppm}$, incubation time: $10 \mathrm{~min}, \mathrm{n}=3$.

linearization and white background normalization routines (Fig. S13). Corrected images were then converted into the HSV and CMYK color spaces from RGB color space for further analysis. Representation of the colors of the MIP samples on the HSV color wheel as shown in Fig. 10b clearly indicates the reason behind the use of S component of HSV and $\mathrm{M}$ component of CMYK color models. $\mathrm{H}$ and $\mathrm{S}$ coordinates represent the color itself and the degree at which the color dominates, respectively [83]. The distance from the origin which corresponds to the S coordinate changes depending on the analyte concentration within a narrow angular range around the origin which corresponds to the $\mathrm{H}$ coordinate. The mean of the $\mathrm{H}$ coordinates $\left(308^{\circ}\right)$ is fairly close to the coordinate of the magenta color $\left(300^{\circ}\right)$ which is plausible since BR9 is involved in the manufacture process of magenta dye [2]. Both S and M channel intensity plots of the MIP samples exhibit discernable sigmoidal responses against the analyte concentration (Fig. S14). On the other hand, the NIP samples possess non-zero $S$ and $M$ values which is attributed to the non-specific binding of BR9 molecules (Fig. S15). The difference between the channel intensities of the MIP and NIP samples were taken as sensor response signals which are denoted as $\Delta S$ and $\Delta M$. The calibration curves were obtained by fitting a sigmoid function, $f(x)$ given by Eq. (11) to the experimental signal data [56]:

$f(x)=a+\frac{b}{1+e^{\frac{x-c}{d}}}$

where $\mathrm{x}$ is the common logarithm of the concentration in units of ppm, and $a, b, c$ and $d$ are the fitting parameters (Table 2). The limit of detection levels and analytical ranges were calculated according to the IUPAC criteria. According to the analysis results, $\Delta M$ signal shows better correlation with the analyte concentration and provides lower detection limit than $\Delta S$ signal (Fig. 10). The sensor system allows the detection of BR9 dye at sub-ppm level which is below the tolerance threshold of 1 ppm [2].

\subsubsection{Binding studies in real samples}

The binding performance of MIP grafted PET film in real samples was evaluated by using natural, tap and industrial wastewater samples (Fig. 11). The binding capacity in ultrapure water was $4.22 \mu \mathrm{g} . \mathrm{g}^{-1}$ at a feed concentration of $1.0 \mathrm{ppm}$. When evaluated using real water samples, a measurable decrease in binding was noted due to interferences caused by chemical or biological ingredients of real matrixes [84]. Still, the specific binding regions of thin MIP layer recognize BR9 and absorb it significantly in real samples too, which provides assurance that a template concentration as low as $1.0 \mathrm{ppm}$ could be determined in a real matrix by the sensor platform via molecular recognition.

\section{Conclusion}

In this work a novel PET surface with a thin (ca. $100 \mathrm{~nm}$ ) MIP layer for the recognition of a model textile dye (BR9) was developed via RAFT-mediated graft polymerization. We believe that immobilizing BP covalently onto a substrate, and then activating it thermally to yield surface radicals is a very powerful tool to modify surface properties of various supporting substrates and tailor them to specific needs. Under optimal conditions, the MIPs showed good selectivity for the target dye versus its analogs and had a sub-ppm LoD, good accuracy and precision for detecting BR9 in aqueous samples. The study further suggests a very promising smartphone-based colorimetric sensor platform for on-site detection of a variety of analytes with chromophores in wastewaters in sub-ppm concentrations. We believe that this study has the potential to contribute to the acceleration of the state-of-the-art technologies aiming to mitigate if not eliminate the use of conventional stationary laboratory equipment for determination of similar water pollutants.

\section{Acknowledgment}

We would like to thank Dr. Songül Karaaslan Aksu from Rudolf GmbH (Bursa, Turkey) for her assistance in supplying industrial wastewater sample.

\section{Appendix A. Supplementary data}

Supplementary material related to this article can be found, in the online version, at doi:https://doi.org/10.1016/j.snb.2019.126653.

\section{References}

[1] F. Deniz, S. Karaman, Removal of basic red 46 dye from aqueous solution by pine tree leaves, Chem. Eng. J. 170 (2011) 67-74.

[2] M.V. Foguel, X.A. Ton, M.V.B. Zanoni, M.P. Sotomayor, K. Haupt, B.T. Bui, A molecularly imprinted polymer-based evanescent wave fiber optic sensor for the detection of basic red 9 dye, Sens. Actuators B Chem. 281 (2015) 222-228.

[3] T.N.V. Souzo, S.M.L. Carvalho, M.G.A. Vieira, M.G.C. Silva, D.S.B. Brasil, Adsorption of basic dyes onto activated carbon: experimental and theoretical investigation of chemical reactivity of basic dyes using dft-based description, Appl. Surf. Sci. 84 (2018) 662-670.

[4] C. Wang, C. Feng, Y. Gao, X. Ma, Q. Wu, Z. Wang, Preparation of a graphene-based magnetic nanocomposite for the removal of an organic dye from aqueous solution, Chem. Eng. J. 173 (2011) (2011) 92-97.

[5] P. Qin, Y. Yang, X. Zhang, J. Niu, H. Yang, S. Tian, J. Zhu, M. Lu, Highly efficient, rapid and simultaneous removal of cation dyes from aqueous solution using monodispersed mesoporous silica nanoparticles as the adsorbent, Nanometarials 8 (2017) (2017) 1-14.

[6] L. Chen, X. Wang, W. Lu, X. Wu, J. Li, Molecular imprinting: perspectives and applications, Chem. Soc. Rev. 45 (2016) 2137-2211.

[7] M. Esteban, Molecularly imprinted polymers: new molecular recognition materials for selective solid-phase extraction of organic compounds, Fred J. Anal. Chem. 370 (2001) 795-802.

[8] Y. Saylan, F. Yılmaz, E. Özgür, A. Derazshamshir, H. Yavuz, A. Denizli, Molecular imprinting of macromolecules for sensor applications, Sensor 17 (2017) 898-928.

[9] K. Haupt, Molecularly imprinted polymers in analytical chemistry, Analyst 126 (2001) 747-756.

[10] S. Beyazit, B. Sum-Bui, K. Haupt, C. Gonzato, Molecularly imprinted polymer nanomaterials and nanacomposites by contoleed/living radical polymerization, Prog. Polym. Sci. 62 (2016) 1-21.

[11] M. Söylemez, O. Güven, M. Barsbay, Method for preparing a well-defined molecularly imprinted polymeric system via radiation-induced RAFT polymerization, Eur. Polym. J. 103 (2018) 21-30.

[12] S. Boonpangrak, M.J. Whitcombe, V. Prachayasittikul, K. Mosbach, L. Ye, Preparation of molecularly imprinted polymers using nitroxide-mediated living radical polymerization, Biosens. Bioelectron. 22 (2006) 349-354.

[13] D. Vaughan, S.P. Sizemore, M.E. Byrne, Enhancing molecularly imprinted polymer binding properties via controlled/living radical polymerization and reaction analysis, Polymer 48 (2007) 74-81.

[14] Y. Zu, X. Zhang, H. Guo, Zhang, Preparation of molecularly imprinted polymers via atom transfer radical "bulk" polymerization, J. Polym. Sci. A Polym. Chem. 48 (2010) 532-541.

[15] J. Liu, W. Wang, Y. Xie, Y. Huang, Y. Liu, X. Liu, R. Zhao, G. Liu, Y. Chen, A novel polychloromethylstyrene coated superparamagnetic surface molecularly imprinted core-shell nanoparticle for bisphenol A, J. Mater. Chem. 21 (2011) 9232-9238.

[16] X. Wei, X. Li, S.M. Husson, Surface molecular imprinting by atom transfer radical polymerization, Biomacromolecule 6 (2005) 1113-1121.

[17] G. Pan, Y. Ma, Y. Zhang, X. Guo, C. Li, H. Zhang, Controlled synthesis of watercompatible molecularly imprinted polymer microspheres with ultrathin hydrophilic polymer shells via surface-initiated reversible addition-fragmentation chain transfer polymerization, Soft Matter 7 (2011) 8428-8439. 
[18] H.J. Wang, W.H. Zhou, X.F. Yin, Z.X. Zhuang, H.H. Yang, X.R. Wang, Template synthesized molecularly imprinted polymer nano-tube membranes for chemical separations, J. Am. Chem. Soc. 128 (2006) 15954-15955.

[19] H. Zhang, J. Jiang, H. Zhang, Y. Zhang, P. Sun, Efficient synthesis of molecularly imprinted polymers with enzyme inhibition potency by the controlled surface imprinting approach, ACS Macro Lett. 2 (2013) 566-570.

[20] L. Yang, Z. Fan, T. Wang, W. Cai, M. Yang, P. Jiang, M. Zhang, X. Dong, Preparation of a pyrazosulfuron-ethyl imprinted polymer with hydrophilic external layers by reversible addition-fragmentation chain transfer precipitation and grafting polymerization, Anal. Lett. 44 (2011) 2617-2632.

[21] X. Li, M. Zhou, M. Turson, S. Lin, P. Jiang, X. Dong, Preparation of clenbuterol imprinted monolithic polymer with hydrophilic outer layers by reversible additionfragmentation chain transfer radical polymerization and its application in the clenbuterol determination from human serum by on-line solid-phase extraction/ HPLC analysis, Analyst 138 (2013) 3066-3074.

[22] M.M. Titirici, B. Sellergren, Thin molecularly imprinted polymer films via reversible addition-fragmentation chain transfer polymerization, Chem. Mater. 18 (2006) 1773-1779.

[23] K. Haupt, A.V. Linares, M. Bompart, B. Sum Bui, Molecularly imprinted polymers, Topp Curr. Chem. 325 (2012) (2012) 1-18.

[24] V.S. Piriya, P. Joseph, D. Kiruba, S. Lakshmanan, T. Kinoshita, S. Muthusamy, Colorimetirc sensors for rapid detection of various analytes, Mat. Sci. Eng. C 78 (2017) 1231-1245.

[25] T. Ye, W. Yin, N. Zhu, M. Yuan, H. Cao, Z. Gou, X. Wang, H. Zhu, R.F. Xu, Colorimetric detection of pyrethroid metabolite by using surface molecularly imprinted polymer, Sens. Actuators B Chem. 254 (2018) 417-423.

[26] L. Feng, C. Musto, J. Kemling, S. Lim, W. Zhong, K.S. Suslick, Colorimetric sensor array for determination and identification of toxic industrial chemicals, Anal. Chem. 82 (2011) 9433-9440.

[27] J. Courbat, D. Briand, J. Damon-Lacoste, J. Wollenstein, N.F. Rooij, Evaluation of $\mathrm{pH}$ indicator-based colorimetric films for ammonia detection using optical waveguides, Sens. Actuators B Chem. 143 (2009) (2009) 62-70.

[28] H. Xiao-Wei, Z. Xiao-Bo, S. Yonh, Z. Jie-Wen, Colorimetric sensor arrays based on chemo-responsive dyes for food odor visualization, Trends Food Sci. 8 (2018) (2018) 90-107.

[29] Q. Kong, Y. Wang, L. Zhang, J. Yu, A novel microfluidic paper-based colorimetric sensor based on molecularly imprinted polymer membranes for highly selective and sensitive detection of bisphenol A, Sens. Actuators B Chem. 243 (2017) 130-136.

[30] S. Feng, Y. Hu, L. Ma, X. Lu, Development of molecularly imprinted polymerssurface-enhanced Raman spectroscopy/colorimetric dual sensor for determination of chlorpyrifos in apple juice, Sens. Actuators B: Chem. 241 (2017) 750-757.

[31] T.A. Sergeyeva, H. Matuschewski, S.A. Piletsky, J. Bendig, U. Schedler, M. Ulbricht, Molecularly imprinted polymer membranes for substance- selective solid-phase extraction from water by surface photo-grafting polymerization, J. Chromatogr. A 907 (2001) (2001) 89-99.

[32] S. Piletsky, E. Piletska, A. Bossi, N. Turner, A. Turner, Surface functionalization of porous polypropylene membranes with polyaniline for protein immobilization, Biotech. Bioeng. 82 (2003) 86-92.

[33] C.J. Stephenson, K.D. Shimizu, Colorimetric and fluorometric molecularly imprinted polymer sensors and binding assays, Poly. Inter. 56 (2007) 482-488.

[34] Y. Zhang, J. Zhang, Q. Liu, Gas sensors based on molecular imprinting technology, Sensors 17 (2017) 1567-1581.

[35] M.H. Schneider, Y. Tran, P. Tabeling, Benzophenone absorption and diffusion in poly(dimethylsiloxane) and its role in graft photo-polymerization for surface modification, Langmuir 27 (2011) 1232-1240.

[36] L. Liu, M. Ren, W. Yang, Preparation of polymeric janus particles by directional UVinduced reactions, Langmuir 25 (2009) 11048-11053.

[37] D. He, M. Ulbricht, Surface-selective photo-grafting on porous polymer membranes via a synergist immobilization method, J. Mater. Chem. 16 (2006) 1860-1868.

[38] Y. Ren, Y. Gu, Q. Zeng, Y. Zhang, UV-induced surface grafting polymerization for preparing phosphorus-containing flame retardant polyacrylonitrile fabric, Eur. Polym. J. 94 (2017) (2017) 1-10.

[39] H. Yu, Y. Cao, G. Kang, J. Liu, M. Li, Q. Yuan, Enhancing antifouling property of polysulfone ultrafiltariton membrane by grafting zwitterionic copolymer via UVinitiated polymerization, J. Membr. Sci. 342 (2009) 6-13.

[40] W. Yang, B. Ranby, Radical living graft polymerization on the surface of polymeric materials, Macromolecules 29 (1996) 3308-3310.

[41] Q. Wang, L. Liu, W. Yang, A novel and facile approach for preparing composite core-shell particles by sequentially initiated grafting polymerization, Polymer 48 (2007) 6581-6588.

[42] C. Barner-Kowollik, Handbook of RAFT Polymerization, Wiley, 2007.

[43] Y. Liu, T. He, C. Gao, Surface modification of poly (ethylene terephthalate) via hydrolysis nd layer-by-layer assembly of chitosan and chondroitin sulfate to construct cytocompatible layer for human endothelial cells, Colloid Surf. B Biointer. 46 (2005) 117-126.

[44] V. Korolkov, A.A. Mashentseva, O. Güven, D.T. Niyazova, M. Barsbay, M.V. Zdorovets, The effect of oxidizing agents/systems on the properties of tracketched PET membranes, Polym. Degrad. Stabil. 107 (2014) 150-157.

[45] S. Schmidt, W. Hanqing, P. Daniel, S. Mosca, L. Hartmann, Probing mutivalency in ligand-receptor mediated adhesion of soft, biomimetic interfaces, Beilstein J. Org. Chem. 11 (2015) 720-729.

[46] H. Hennig, C. Borcherding, S. Jaeger, C. Hatami, A. Würth, K. Hoffmann, T. Thiele, U. Schedler, U. Resch-Genger, Scope and limitations of surface functional group quantification methods: exploratory study with poly (acrylic acid)-grafted microand nanoparticles, J. Am. Chem. Soc. 134 (2012) 8268-8276.

[47] M. Baumi, W.J. Brittain, Snyhesis of polymer brushes on silicate substrates via reversible addition fragmentation chain transfer technique, Macromolecules 35 (2002) 610-615.

[48] N. Lopez-Ruiz, V. Curto, M.M. Erenas, F. Benito-Lopez, D. Diamond, A.J. Palma, L.F. Capitan-Vallvey, Smartphone-based simultaneous ph and nitrite colorimetric determination for paper microfluidic devices, Anal. Chem. 86 (2014) 9554-9562.

[49] Y. Jung, J. Kim, O. Awofeso, H. Kim, F. Regnier, E. Bae, Smartphone-based colorimetric analysis for detection of saliva alcohol concentration, Appl. Opt. 54 (2015) 9183-9189.

[50] M.Y. Jia, Q.S. Wu, H. Li, Y. Zhang, Y.F. Guan, L. Feng, The calibration of cellphone camera-based colorimetric sensor array and its application in the determination of glucose in urine, Biosens. Bioelectron. 74 (2015) 1029-1037.

[51] Y. Mutlu, V. Kılıç, G.K. Özdemir, A. Bayram, N. Horzum, M.E. Solmaz, Smartphonebased colorimetric detection via machine learning, Analyst 142 (2017) 2434-2441.

[52] A.W. Martinez, S.T. Phillips, G.M. Whitesides, Three-dimensional microfluidic devices fabricated in layered paper and tape, Proc. Natl. Acad. Sci. 105 (2008) 19606-19611.

[53] S. Paciornik, A.V. Yallouz, R.C. Campos, D. Gannerman, Scanner image analysis in the quantification of mercury using spot-tests, J. Braz. Chem. Soc. 17 (2006) $156-161$.

[54] K. Yetisen, J.L. Martinez-Hurtado, A. Garcia-Melendrez, F. Cruz Vasconcellos, C.R. Lowe, A smartphone algorithm with inter-phone repeatability for the analysis of colorimetric tests, Sens. Actuators B Chem. 196 (2014) (2014) 156-160.

[55] L. Shen, J.A. Hagen, I. Papautsky, Point-of-care colorimetric detection with a smartphone, Lab Chip 12 (2012) 4240-4243.

[56] M.M. García, E.D. Erenas, C.A. Marinetto, I. Abad, A.J. Orbe-Paya, A.J. Palma, L.F. Capitán-Vallvey, Mobile phone platform as portable chemical analyzer, Sens. Actuators B Chem. 156 (2011) 350-359.

[57] E. Gao, E. Huddleston, J. White, H. Pant, J. Handa, J. Locklin, Surface grafted antimicrobial polymer networks with high abrasion, ACS Biomat. Sci. Eng. 2 (2016) 1169-1179.

[58] Y. Ma, L. Liu, W. Yang, Photo induced living/controlled surface radical grafting polymerization and its application in fabricating 3-D micro-architectures on the surface of flat/particulate organic substances, Polymer 52 (2011) 4159-4173.

[59] H. Hong, N. Liu, G. Sun, UV-induced graft polymerization of acrylamide on cellulose by using immobilized benzophenone as a photo initiator, Eur. Poly. J. 45 (2009) 2443-2449.

[60] J. Kim, J.A. Hanna, M. Byun, C.D. Santangelo, R.C. Hayward, Designing responsive buckled surfaces by halftone gel lithography, Science 335 (2012) 1201-1205.

[61] T. Brandstetter, S. Boehmer, O. Prucker, E. Bisse, A. Hausen, J. Alt-Moerbe, J.A. Ruehe, Polymer-based DNAbiochip platform for human papilloma virus genotyping, J. Virol. Methods 163 (2010) 40-48.

[62] M.M. Virkar, J. Ling, Z. Locklin, Z. Bao, Oligothiophene based organic semiconductors with cross-linkable benzophenone moieties, Synth. Met. 158 (2008) 958-963.

[63] J. Holland, J.N. Hay, The thermal degradation of PET and analogous polyesters measured by thermal analysis - Fourier transform infrared spectroscopy, Polymer 43 (2002) 1835-1847.

[64] M. Barsbay, Y. Kodama, O. Güven, Functionalization of cellulose with epoxy groups via $\gamma$-initiated RAFT-mediated grafting of glycidyl methacrylate, Cellulose 21 (2014) 4067-4079.

[65] G. Çelik, M. Barsbay, O. Güven, Towards new proton exchange membrane materials with enhanced performance via RAFT polymerization, Polym. Chem. 7 (2016) 701-714.

[66] M. Barsbay, O. Güven, Y. Kodama, Amine functionalization of cellulose surface grafted with glycidyl methacrylate by gamma-initiated RAFT polymerization, Radiat. Phys. Chem. 124 (2016) 140-144.

[67] X. Li, S.M. Husson, Adsorption of dansylated amino acids on molecularly imprinted surfaces: a surface plasmon resonance study, Biosens. Bioelectron. 22 (2006) $336-348$.

[68] H. Li, N. Li, D.J. Chen, Q. Xu, H. Li, J. He, J. Lu, Molecularly impinted magnetic microparticles for the simultaneous detection and extraction of Rhodamine B, Sens. Actuators B Chem. 246 (2017) 286-292.

[69] H. Kalavathy, T. Karthikeyan, S. Rajgopal, L.R.J. Miranda, Kinetic and isotherm studies of $\mathrm{Cu}(\mathrm{II})$ adsorption onto $\mathrm{H}_{3} \mathrm{PO}_{4}$-activated rubber wood sawdust, Colloid Interface Sci. 292 (2005) 354-362.

[70] G.T. Rushton, C.L. Karns, K.D. Shimizu, A critical examination of the use of the Freundlich isotherm in characterizing molecularly imprinted polymers (MIPs), Anal. Chim. Acta 528 (2005) 107-113.

[71] S.H. Cheong, A.E. Rachkov, J.K. Park, K. Yano, I. Karube, Synthesis and binding properties of a non-covalent molecularly imprinted testosterone- specific polymer, J. Polym. Sci. Part A1 36 (1998) 1725-1732.

[72] M. Rampey, R.J. Umpleby, G.T. Rushton, C. Iseman, R. Shah, K.D. Shimizu, Characterization of of the imprint effect and the influence of imprinting conditions on affinity, capacity and heterogeneity in imprinted polymers using the Freundlich isotherm-affinity distribution analysis, Anal. Chem. 76 (2004) 1123-1133.

[73] R.J. Umpleby, S.C. Baxter, Y. Chen, R. Shah, K.D. Shimizu, Characterization of molecularly imprinted polymers with the Langmuir-Freundslich isotherm, Anal. Chem. 73 (2001) (2001) 4584-4591.

[74] X. Yu, H. Liu, J. Diao, Y. Sun, Y. Wang, Magnetic molecularly imprinted polymer nanoparticles for separating aromatic amines azo dyes-synthesis, characterization and application, Sep. Purif. Technol. 204 (2018) 213-219.

[75] M. Söylemez, M. Barsbay, O. Güven, Preparation of well-defined erythromycin imprinted non-woven fabrics via radiation-induced RAFT-mediated grafting, Radiat. Phys. Chem. 142 (2018) 77-81.

[76] X. Lu, Yue, F. Lini, F. Huang, B. Zhang, Z. Lin, Template-synthesized ultra-thin molecularly imprinted polymers membrane for the selective preconcenration of 
dyes, J. Mater. Chem. A 3 (2015) 10959-10968.

[77] V. Vadivelan, K.V. Kumar, Equilibrium, kinetics, mechanism, and process design for the sorption of methylene blue onto rice husk, J. Coll. Interf. Sci. 286 (2005) 90-100.

[78] A. Alenazi, J.M. Manthorpe, P.C.E. Lai, Selectivity enhancement in molecularly imprinted polymers for binding of bisphenol A, Sensors 16 (2016) 1697-1709.

[79] M.C. Foguel, N.T. Pedro, A. Wong, S. Khan, M.V.B. Zanoni, P.T. Sotomayor, Synthesis and evaluation of a molecularly imprinted polymer for selective adsorption and quantification of acid green 16 textile dye in water samples, Talanta 170 (2017) 244-251.

[80] S. Boulanouar, S. Mezzache, A. Combes, V. Pichon, Molecularly imprinted polymers for the determination of organophosphorus pesticides in complex samples, Talanta 176 (2018) 465-478.

[81] F. Duan, C. Chen, X. Zhao, Y. Yang, X. Liu, Y. Quin, Water-compatible surface molecularly imprinted polymers with synergy of bi-functional monomers for enhanced selective adsorption of bisphenol A from aqueous solution, Environ. Sci. Nano 3 (2016) 213-222.

[82] Hu X, Y. Fa, Y. Zhang, G. Da, Q. Cai, Y. Cai, C. Guo, Molecularly imprinted polymer coated solid-phase microextraction fiber prepared bu surface reversible additionfragmentation chain transfer polymerization for monitoring of sudan dyes in chilli tomato sauce and chilli pepper samples, Anal. Chim. Acta 731 (2012) 40-48.

[83] K. Cantrell, M.M. Erenas, I. de Orbe-Payá, L.F. Capitán-Vallvey, Use of the hue parameter of the hue, saturation, value color space as a quantitative analytical parameter for bitonal optical sensors, Anal. Chem. 82 (2010) 531-542.

[84] X. Luo, Y. Zhan, Y. Huang, L. Yang, X. Tu, S. Luo, Removal of water-soluble acid dyes from water environment using a novel magnetic molecularly imprinted polymer, J. Hazard. Mater. 187 (2011) 274-282. 